\title{
The Impact of High-Frequency Repetitive Transcranial Magnetic Stimulation on Fine Motor Functions in Medication-Resistant Major Depression
}

\author{
Didier L. Schrijvers ${ }^{a, b} \quad$ Chris Baeken ${ }^{c, e} \quad$ Rudi De Raedt ${ }^{d} \quad$ Bernard G.C. Sabbe $^{a, b}$ \\ ${ }^{a}$ Collaborative Antwerp Psychiatric Research Institute, University of Antwerp, Faculty of Medicine and \\ Health Sciences, Antwerp, ${ }^{b}$ Psychiatric Hospital Sint-Norbertushuis, Duffel, Departments of ${ }^{C}$ Psychiatry and \\ Medical Psychology and ${ }^{d}$ Experimental Clinical and Health Psychology, Ghent University, Ghent, and \\ eDepartment of Psychiatry, University Hospital UZBrussel, Brussels, Belgium
}

\section{Key Words}

Transcranial magnetic stimulation • High-frequency repetitive transcranial magnetic stimulation - Psychomotor symptoms · Retardation • Major depressive disorder

\begin{abstract}
Objectives: Although high-frequency repetitive transcranial magnetic stimulation (HF-rTMS) over the left dorsolateral prefrontal cortex (DLPFC) has been reported to improve mood symptoms in major depressive disorder (MDD), research on its impact on psychomotor symptoms is scarce. This study assessed the psychomotor effects of 1 and 10 sessions, respectively, of HF-rTMS over the left DLPFC. Methods: Ten HF-rTMS sessions were applied in 21 medication-free MDD patients over a 2-week period. At the beginning, one placebo (sham)-controlled rTMS session was also applied in a cross-over, single-blind design. Psychomotor variables were digitally recorded during completion of a Fitts' task, at baseline, after the first and second real/sham session and at the end point. Results: The total 10-session treatment period resulted in a decrease of depression severity. One HFrTMS session resulted in improvements on the Fitts' task, without a difference between active and sham stimulation, however. No further improvements occurred from session 2
\end{abstract}

\section{KARGER}

Fax +41613061234 E-Mail karger@karger.ch www.karger.com
(C) 2012 S. Karger AG, Basel

0302-282X/12/0664-0252\$38.00/0

Accessible online at:

www.karger.com/nps to session 10. Conclusions: No evidence was provided to link the observed psychomotor improvements to HF-rTMS stimulation, as a practice effect could have impacted the significant psychomotor outcomes. Copyright $\odot 2012$ S. Karger AG, Basel

\section{Introduction}

In recent years, studies investigating the therapeutic efficacy of repetitive transcranial magnetic stimulation (rTMS) for major depressive disorder (MDD) have exponentially increased [1-3]. A growing body of evidence has been published indicating that depressed patients can be successfully treated with high-frequency (HF)-rTMS when this is administered on the left dorsolateral prefrontal cortex (DLPFC) [4-6]. Recent meta-analytic studies support the antidepressant efficacy of this technique in treatment-resistant depressed patients when stimulation periods are long enough (e.g. $>2$ weeks) $[2,3]$. Besides the treatment duration, the frequency and which side is

D.L.S. and C.B. contributed equally to this work. 
stimulated should also be taken into account when evaluating the efficacy of rTMS [3].

Most rTMS studies focus on the reduction in mood symptoms in MDD, but the effects of rTMS on psychomotor functioning have been rarely explored.

Notwithstanding, cognitive dysfunctions and psychomotor retardation have also been determined as core features of episodes of MDD [7-9]. Psychomotor slowing appears to be a strong diagnostic marker for MDD with melancholic features. As psychomotor retardation is one of the key symptoms of the melancholic subtype of depression, it would be very appropriate to investigate psychomotor changes following rTMS treatment in a cohort of melancholic patients. From a neurobiological perspective, psychomotor retardation in major depression - and especially the melancholic subtype - has been linked to a hypodopaminergic state [7,9]. Moreover, prefrontal rTMS has been found to influence striatal dopaminergic activity $[10,11]$. Furthermore, a higher level of psychomotor retardation has been associated with reduced metabolic activities in the left DLPFC suggesting an important role of this cortical area on psychomotor functioning [9]. Therefore, given the possible neurobiological and clinical implications, a thorough investigation of the psychomotor effects of rTMS in MDD is warranted.

A limited number of studies have already examined the neurocognitive effects of rTMS in MDD $[12,13]$, but even fewer studies have examined the impact of rTMS on psychomotor performance in MDD. Applying the motor agitation and retardation scale (MARS), Hoeppner et al. [14] observed a reduction in psychomotor retardation following 10 HF-rTMS sessions over the left DLPFC. They could only demonstrate a trend in the reduction of psychomotor agitation, but not for retardation in MDD following 15 left prefrontal HF-rTMS sessions [15]. Finally, Baeken et al. [16] demonstrated the positive effect of 10 sessions of HF-rTMS on psychomotor slowing as measured by means of the Salpêtrière Retardation Rating Scale (SRRS).

Given the limited number of studies on this subject and their divergent results, this study further investigated the psychomotor effects of HF-rTMS in MDD, applying the Fitts' task. This computerized task is an objective and reliable method to assess fine motor activity, and is generally considered to be a rater-independent and more objective measurement method than the more subjective rating scales [9]. The Fitts' task has been widely used in the research into psychomotor symptoms in MDD [1719]. This fine motor task requires a precise sensorimotor programming, initiation and execution of the muscle commands [17-19]. One HF-rTMS session with parameters comparable to rTMS treatment for depression has been reported to affect this psychomotor functioning in healthy subjects [20].

Consequently, this study aims to further explore the psychomotor effects of HF-rTMS over the left DLPFC in a sample of medication-resistant MDD patients, applying the Fitts' task. To evaluate the effect of a single stimulation on psychomotor functioning, we assessed the effects of one sham-controlled session of HF-rTMS delivered on the left DLPFC, in a single-blind placebo controlled cross-over design. To examine the effect of HF-rTMS treatment, the effect on the Fitts' task was assessed after 10 such sessions spread over a period of 2 weeks of treatment. To ascertain that the melancholic depressed patients displayed decreased psychomotor speed, their baseline Fitts' measurements were compared to an ageand gender-matched control group.

As mentioned above, few studies on psychomotor effects in MDD have been executed up to now, psychomotor retardation has been associated with DLPFC hypofunction and a hypodopaminergic state and rTMS has been supposed to exert an impact on the dopaminergic system. Together with the limited existing evidence mentioned above, all these findings led us to hypothesize that an improvement in psychomotor functioning could be expected after HF-rTMS treatment, particularly in our treatment responder group.

\section{Methods}

\section{Patients}

Our group consisted of 21 medication-free unipolar depressed patients of the melancholic subtype (female:male =13:8; age 44.7 \pm 10.3 years). Psychiatric disorders were assessed using the MiniInternational Neuropsychiatric Interview [21]. Because concomitant personality disorders were not part of the exclusion criteria no formal diagnostic screening on axis II diagnosis was performed. Severity of depression was assessed with the 21-item Beck Depression Inventory (BDI) [22] and the 17-item Hamilton Depression Scale (HDRS) [23]. The HDRS was administered by an experienced psychiatrist, not related to the study. Eleven participants were current in-patients during HF-rTMS treatment. Treatment resistance was assessed with criteria according to Thase and Rush [24]. All were right-handed and considered at least stage-III treatment-resistant: they had had a minimum of 2 unsuccessful trials of SSRI/NSRI treatment and 1 failed trial with tricyclic antidepressants as described by Rush et al. [25]. Because concomitant antidepressant treatment can confound outcome results, all patients went through a 2 -week antidepressant washout before entering the study (this was over 3 weeks if they were on fluoxetine). Where necessary, patients were kept on a steady dose of their 'somatic' medications. During the washout period, patients 


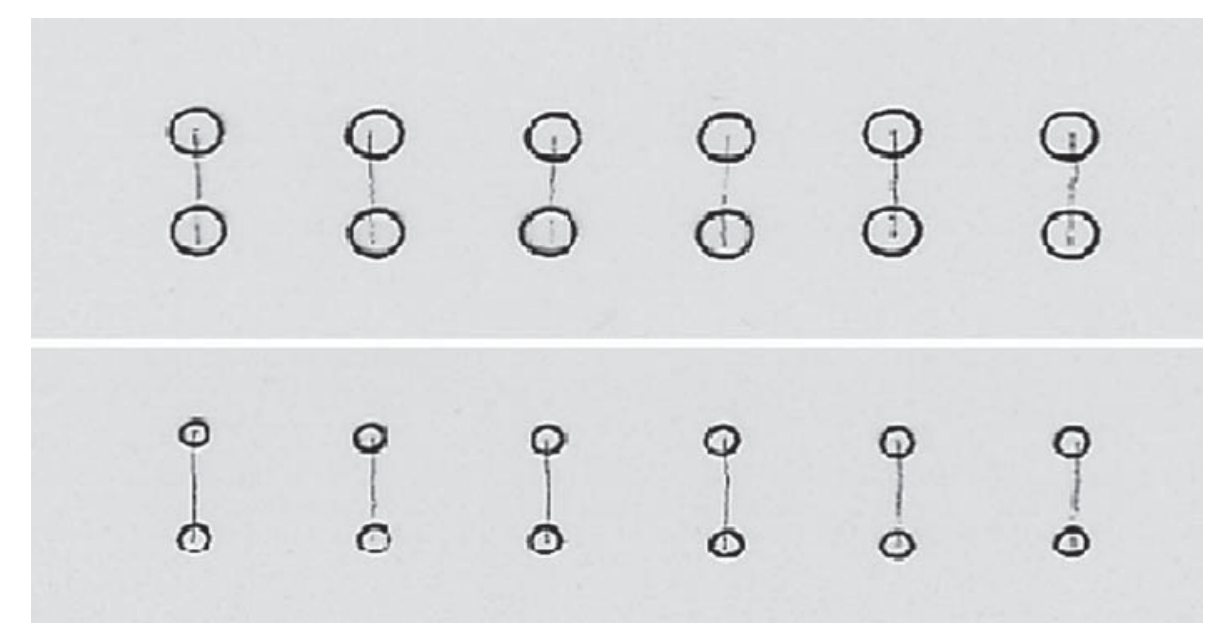

Fig. 1. Fitts' task.

had contact with their physicians on a regular basis. Only habitual benzodiazepine agents were allowed: 1 subject took alprazolam (1 mg), 1 took clonazepam (0.5 mg), 1 took flunitrazepam (1 mg) and 1 took alprazolam (1 mg) and flurazepam (27 mg). During our stimulation protocol, all psychopharmacological changes were considered as dropout from the study. Additionally, no changes of habitual somatic treatment were allowed. All subjects underwent physical, neurological (MRI, EEG) and psychiatric examinations.

Exclusion criteria were: a current or past history of epilepsy, neurosurgery, having metal or magnetic objects in the brain and being pregnant. Patients with suicide attempts during their current depressive episode or alcohol/drug dependence and/or abuse were not included.

A group of 28 healthy controls (mean age: $40.82 \pm 6.93$; male:female ratio $=11 / 17$ ) was included matched for sex and age with the patient group ( $\mathrm{p}=0.20$ and $\mathrm{p}=0.77$, respectively). These controls were recruited in the context of a previous research project on psychomotor functioning in major depression [26]. This study was part of a larger project investigating the influence of HF-rTMS on different neurocognitive markers. The study was consistent with the latest version of the Helsinki Declaration and was approved by the ethics committee of the University Hospital (UZBrussel) of the Vrije Universiteit Brussel. All subjects gave written informed consent.

\section{Psychomotor Assessments}

For the objective psychomotor measurement, all participants carried out a Fitts' task (see fig. 1) using a pressure-sensitive inking ballpoint pen on sheets of paper placed on a digitizer that was connected to a personal computer. The Fitts' task is a computerized fine motor task that has been designed especially to evaluate sensorimotor programming, initiation and execution of muscle commands without requiring higher-order cognitive processes [17-19]. In this task, subjects had to connect two vertically placed circles, depicted on a normal sheet of paper, by drawing a line of about $1 \mathrm{~cm}$. They were instructed to start in the middle of the top circle and to end in the middle of the lower circle. Per trial, 6 lines had to be drawn. The accuracy of movement was varied by changing the circle diameter from $0.50 \mathrm{~cm}$ in trials 1 and 4 (fig. 1, upper part) to $0.25 \mathrm{~cm}$ in trials 2 and 3 (fig. 1, lower part). Movement time was recorded, i.e. the time between the starting and completion of each separate line-drawing movement. The movement times of inaccurate line drawings, i.e. when the line was drawn from the lower to the upper circle and/or when the start/end point of the connecting line was situated outside the circle diameter, were not included in the analyses.

In addition, visual analog scales (VAS) were used to examine subjective mood changes: subjects were asked to rate their mood on 5 horizontal 100-mm VAS in order to detect subtle changes in feelings of 'depression', 'fatigue', 'tension', 'anger' and 'vigor'. The minimum score on each VAS subscale is 0 and the maximum score is 100. Right-handedness was assessed with the Van Strien questionnaire [27].

\section{Design and rTMS Procedure}

Patients underwent 10 sessions of HF-rTMS on the left DLPFC within a period of 2 weeks. At the beginning of this open-treatment trial, each subject also received 1 placebo (sham) HF-rTMS stimulation session, separated from the first active stimulation session by 1 day. This phase was a placebo-controlled cross-over, single-blind design allowing examination of single session, specific rTMS effects in MDD patients.

Potential mood changes were assessed before (Tpre), immediately after (Tpost) and $30 \mathrm{~min}$ after (Tpost30) terminating the first rTMS (real/sham) session, using VAS scales.

The antidepressant effects of 2 weeks of rTMS treatment were investigated by assessing the HDRS and BDI at baseline and at end point, i.e. after the eleventh stimulation session (1 of which was a placebo session). The Fitts' task was applied before (Tpre) immediately after (Tpost) terminating the first and second rTMS (real/ sham) session, and at the end of the rTMS treatment period (Tpost-treatment i.e. after the last session).

Because this study was part of a larger project investigating the influence of HF-rTMS on different neurocognitive markers, additional tasks were also administered that were not used for the current study purposes. Measures were always presented in the same order for all participants.

Patients were kept unaware of the type of stimulation; they wore earplugs and were blindfolded. Importantly, all patients 
were stimulated on all occasions within the same time schedule, i.e. between $10 \mathrm{am}$ and noon.

For the application of rTMS, we used a Magstim high-speed magnetic stimulator (Magstim Company Limited, Wales, UK), connected to a figure-of-8-shaped coil. Before each application, the motor threshold of each individual was determined using electromyography. A stimulation intensity of $110 \%$ of the subject's motor threshold of the right abductor pollicis brevis muscle was used. In order to accurately target the left DLPFC (Brodmann area 9/46), the precise stimulation site and position of the coil were determined using MRI nonstereotactic guidance (Philips Intera, Best, The Netherlands). Perpendicular to this point, the precise stimulation site on the skull was marked and stimulated [28]. In each $\mathrm{HF}(10 \mathrm{~Hz})$ stimulation session, subjects received 40 trains of 3.9-second duration, separated by an intertrain interval of 26.1 s. Each session, therefore, lasted $20 \mathrm{~min}$ (1,560 pulses per session). For the sham condition, the coil was held at an angle of $90^{\circ}$, with one edge only resting on the scalp. The International Society of Transcranial Magnetic Stimulation safety guidelines were followed $[29,30]$.

\section{Statistics}

All results were analyzed using the SPSS for Windows 16.0 software package. Statistical analyses were performed using ANOVA's. The significance level was set at $\mathrm{p}<0.05$ for all analyses. Baseline and end-point psychomotor outcomes in the patient group were compared with the outcomes of an age- and gendermatched control group that participated in a previous research project in which psychomotor measurements were registered [26]. Separate analyses were conducted to investigate the single-session effect ( 1 active/sham session) and the treatment effect (10 active sessions) of HF-rTMS.

Regarding the single-session effects, we used separate $3 \times 2$ ANOVA's with the VAS mood scales as dependent variables and Session (Tpre, Tpost or Tpost30) and Stimulation (active, sham) as within-subject factors. With regard to the analyses for the single-session effects on the Fitts' task, a $2 \times 2 \times 2$ factorial design was used with Stimulation (active, sham), Session (Tpre, Tpost) and Complexity (small circles, large circles) as within-subject factors.

Subsequently, overall improvement in HDRS and BDI scores between the baseline and final assessment were analyzed using paired Student $t$ tests over the whole group. Clinical response was defined as a $50 \%$ reduction of the baseline HDRS score.

A similar $2 \times 2 \times 2$ design was applied for the psychomotor outcomes related to the 10 -session treatment with Session (Tpre, Tpost-treatment) and Complexity (small circles, large circles) as within-subject factors, and Treatment Response (responders, non-responders) as a between-group factor.

\section{Results}

\section{Baseline Psychomotor Outcomes}

A comparison of the baseline Fitts' outcomes of the patient group with those of an age- and gender-matched control group [mean movement time (MT) $255 \mathrm{~ms}$ and standard deviation (SD) 0.25] pointed to a significantly

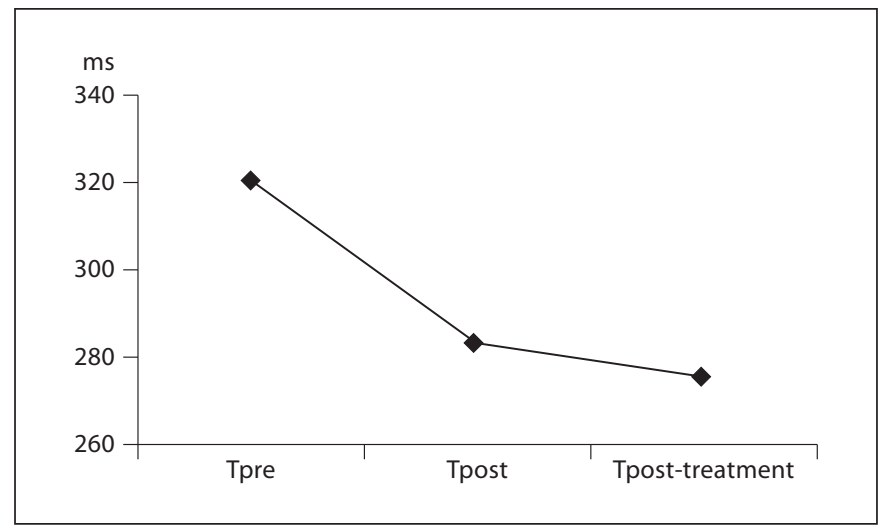

Fig. 2. Mean movement times (in ms) of the patient group from baseline to end point for the Fitts' task.

slower performance of the patients (mean MT $321 \mathrm{~ms}$, SD $0.12, \mathrm{t}=2.21$ and $\mathrm{p}<0.05)$.

\section{Single Session Effects}

Mood. VAS analyses were conducted on 20 patients because 1 subject had numerous missing values. The separate ANOVAs did not reveal any significant effect, neither for the main effect of Stimulation (all F values $<1.33$ ) or Session (all F values <2.45), nor for the Stimulation by Session interaction (all F values $<1.09$ ). The only exception was the VAS depression subscale that showed a main effect of Session $[\mathrm{F}(2,18)=8.38, \mathrm{p}<0.01]$ with the scores slightly decreasing from Tpre (6.46) over Tpost (5.12) to Tpost30 (5.74), without a significant Stimulation by Session interaction $[\mathrm{F}<1]$, however.

\section{Psychomotor Variables}

As demonstrated in previous studies, a smaller diameter of the circles resulted in significantly higher MTs $[\mathrm{F}(1,20)=94.6, \mathrm{p}<0.001]$. Moreover, a significant main effect of Session was found with the MTs improving from prestimulation to poststimulation $[\mathrm{F}(1,20)=5.68, \mathrm{p}<$ 0.05; Tpre: $309 \mathrm{~ms}$, Tpost: $284 \mathrm{~ms}$ ]. However, neither the Stimulation by Session interaction nor any of the other interactions was significant [all Fs $<1$ ], with the exception of the Complexity by Session interaction $[\mathrm{F}(1,20)=3.91$, $\mathrm{p}<0.1]$. These results indicate that the first rTMS session did improve fine motor performance but no difference could be demonstrated between active (Tpre: $306 \mathrm{~ms}$; Tpost: $279 \mathrm{~ms}$ ) or sham (Tpre: $311 \mathrm{~ms}$; Tpost: $287 \mathrm{~ms}$ ) stimulation within that time frame. It should be noted that patients remained slower than healthy controls after the first stimulation session $(\mathrm{F}=3.14, \mathrm{p}<0.1)$. 


\section{Treatment Effects}

Mood. Mean HDRS scores before entering the study were $25.24(\mathrm{SD}=3.9)$ and mean $\mathrm{BDI}$ scores were 33.82 $(\mathrm{SD}=12.19)$, indicating severe depression. The overall patient group demonstrated a significant improvement in HDRS and BDI scores between the baseline and final assessment (final HDRS: $15.35, \mathrm{t}=5.61, \mathrm{p}<0.001$; final BDI: $25.27, \mathrm{t}=2.76, \mathrm{p}<0.05)$. Eleven patients $(52 \%)$ were considered clinical nonresponders and the other $10(48 \%)$ clinical responders.

\section{Psychomotor Variables}

A significant psychomotor improvement was observed from baseline to end point [Tpre: $321 \mathrm{~ms}$, Tpost-treatment: $276 \mathrm{~ms} ; \mathrm{F}(1,19)=4.22$, $\mathrm{p}<0.05$; see fig. 2], as well as the well known effect of Complexity $[\mathrm{F}(1,19)=80.88$, $\mathrm{p}<0.001]$. Neither the main effect of, nor any interactions with Treatment Response were significant [Fs < 2.12].

A comparison of the end-point Fitts' outcomes of the patient group (mean MT: $276 \mathrm{~ms}$ ) with the baseline data of the control group (mean MT: $255 \mathrm{~ms}$ ) revealed no significant differences in psychomotor performance $(\mathrm{t}=$ $0.78, \mathrm{p}=0.44$ ).

Note that no further substantial psychomotor improvements occurred between session 2 (which was an HF-rTMS or a sham session) and session 10 (last HF-rTMS session): $\mathrm{F}<1, \mathrm{p}=0.36$. This could imply that the observed psychomotor improvements are obtained between the first and the second psychomotor assessment (following 1 sham/ rTMS stimulation), which further underscores the previously mentioned impact of there being a practice effect.

Additional correlational analyses were conducted to further investigate the association between the clinical and psychomotor variables. No significant correlations could be observed, either between the absolute difference of the baseline and end-point rTMS psychomotor variable and the absolute difference in HDRS/BDI scores, or between the proportional changes of mentioned variables (all $\mathrm{r}$ values $<0.34$, all $\mathrm{p}$ values $>0.15$ ). In this context, it should also be mentioned that the Fitts' outcomes at baseline and end point did not correlate with the duration of the current episode or the stage of treatment resistance (all $\mathrm{r}$ values $<0.19$ and all $\mathrm{p}$ values $>0.4$ ).

\section{Discussion}

This study explored the clinical and psychomotor effects of 1 sham-controlled and 10 active HF-rTMS sessions, respectively, in treatment-resistant MDD applying clinical rating scales and an objective psychomotor assessment method, i.e. the Fitts' task.

At baseline, MDD patients performed significantly slower than an age- and gender-matched control group, which has also been demonstrated repeatedly in previous studies from our research group [9]. The total patient sample manifested a clear improvement in depression severity following 10 active rTMS sessions with approximately half of the patient sample manifesting a clear clinical response, as determined with a $50 \%$ decrease of the initial HDRS scores, which is in line with previous HFrTMS treatment studies in MDD [31]. Regarding the VAS, one HF-rTMS session did decrease the depression subscale scores, irrespective of the stimulation type (active or sham), however. Scores on the other VAS subscales did not significantly change following one session.

Focussing on the effects of one HF-rTMS-session, psychomotor improvements were observed on the Fitts' task, but did not reveal a difference between active and sham stimulation. The analyses investigating the total treatment period of $10 \mathrm{HF}-\mathrm{rTMS}$ sessions did point to a better end-point performance on the Fitts' task, but these psychomotor improvements are likely due to a practice effect on the task. Indeed, the psychomotor improvements observed after session one emerged irrespective of sham or active HF-rTMS stimulation, and no further improvements occurred from session 2 to session 10. Notwithstanding, it should be mentioned that for every assessment session, patients were given the opportunity to practice with and get used to the task, before the proper recordings started.

Besides the mentioned practice effect, it might be possible that due to certain nonspecific aspects related to the rTMS procedure - such as its impressive name, its discomfort and its sophisticated-looking equipment - a placebo effect could have influenced the one-session outcomes [32]. In addition, we cannot totally rule out the possible impact of our sham control condition: although this was performed at a $90^{\circ}$ angle, ensuring minimal stimulation of the DLPFC, it could still be possible that a partially active placebo was used [33].

Whereas the current study mainly focused on fine motor performance, it would also be interesting to obtain more knowledge on the effects of rTMS on gross motor performance. However, the relationship between gross and fine motor performance in MDD has not yet been elucidated and it is not clear whether MDD patients with fine motor dysfunction are affected to the same degree in their gross motor performance [9]. Therefore, in order to investigate the impact of rTMS on gross motor perfor- 
mance, a similar rTMS design should be executed with assessment methods of gross motor activity such as actometric measurements.

In addition, most previous studies have examined the psychomotor effects of rTMS treatment applying clinical rating scales. Hoeppner et al. [14] reported a significant improvement of baseline MARS-rated psychomotor retardation after 10 HF-rTMS sessions spread over 2 weeks, for both a group treated with $20 \mathrm{~Hz}$ HF-rTMS and a group treated with $1 \mathrm{~Hz}$ HF-rTMS, whereas a sham group did not manifest any psychomotor improvement. In another study, however, Hoeppner et al. [15] could not demonstrate a beneficial effect of left prefrontal $10 \mathrm{~Hz}$ HF-rTMS treatment during 15 days on MARS-assessed psychomotor retardation. Instead, they found nearly significant reductions in the agitation symptoms. Very recently, Baeken et al. [16] reported a decrease in SRRS scores after 10 sessions of $10 \mathrm{~Hz}$ HF-rTMS over the left DLPFC. In this context, it needs to be mentioned that rating scales and the applied psychomotor assessment method substantially differ in their duration of observation: rating scales are based on prolonged clinical observations whereas experimental tasks only capture fine motor performance during task execution [9]. Moreover, rating scales can be rater-dependent whereas the currently used assessment method does not depend on the rater [9].

A limitation of this study could be the number of HFrTMS sessions. Despite rTMS treatment parameters being quite intense, the duration of 2 weeks might be considered as rather short. Indeed, current HF-rTMS treatment protocols stimulate patients daily for 3-6 weeks $[6,34]$.
In addition, as a higher level of treatment resistance in the current depressive episode might be inversely related to clinical outcome, this might to some extent have impacted our fine motor task results [35].

A major strength of this study is that all patients had a sufficient washout period from their antidepressants, whereas in several other studies patients were still taking their current psychotropic medication. Moreover, during the stimulation protocol, no changes to patients' habitual somatic treatment were allowed. On the other hand, although all included patients in our analysis continued with exactly the same benzodiazepine concentrations, the use of benzodiazepines in our sample might have been a confounding variable [19].

Future studies might do well to further objectively investigate fine motor functioning in depressed case samples in association with rTMS treatment. More intensive placebo-controlled rTMS studies are needed to further disentangle the effect of HF-rTMS on the psychomotor system as clinical improvement with these kinds of techniques are reported.

\section{Acknowledgments}

The authors would like to thank all patients for their participation, and Yvonne Maas and Sara Vermeylen for their help in the preparation of the data.

This research was supported by a grant from the Scientific Fund W. Gepts UZBrussel. Preparation of this paper was also supported by the Ghent University Multidisciplinary Research Partnership 'The integrative neuroscience of behavioral control'.

\section{References}

1 Mitchell PB, Loo CK: Transcranial magnetic stimulation for depression. Aust NZ J Psychiatry 2006;40:406-413.

-2 Daskalakis ZJ, Levinson AJ, Fitzgerald PB: Repetitive transcranial magnetic stimulation for major depressive disorder: a review. Can J Psychiatry 2008;53:555-566.

- 3 Dell'osso B, Camuri G, Castellano F, Vecchi V, Benedetti M, Bortolussi S, Altamura AC: Meta-review of meta-analytic studies with repetitive transcranial magnetic stimulation (rTMS) for the treatment of major depression. Clin Pract Epidemiol Ment Health 2011;7:167-177.

4 Gershon AA, Dannon PN, Grunhaus L: Transcranial magnetic stimulation in the treatment of depression. Am J Psychiatry 2003; 160:835-845.
5 Schutter DJ: Antidepressant efficacy of highfrequency transcranial magnetic stimulation over the left dorsolateral prefrontal cortex in double-blind sham-controlled designs: a meta-analysis. Psychol Med 2009;39:65-75.

-6 Fitzgerald PB, Daskalakis ZJ: A practical guide to the use of repetitive transcranial magnetic stimulation in the treatment of depression. Brain Stimul 2012;5:287-296.

7 Sobin C, Sackeim HA: Psychomotor symptoms of depression. Am J Psychiatry 1997; 154:4-17.

$\$ 8$ Dantchev N, Widlöcher DJ: The measurement of retardation in depression. J Clin Psychiatry 1998;59(suppl)14:19-25.

-9 Schrijvers D, Hulstijn W, Sabbe BGC: Psychomotor symptoms in depression: a diagnostic, pathophysiological and therapeutic tool. J Affect Disord 2008;109:1-20.
10 Keck ME, Welt T, Mueller MB, Erhardt A, Ohl F, Toschi N, et al: Repetitive transcranial magnetic stimulation increases the release of dopamine in the mesolimbic and mesostriatal system. Neuropharmacol 2002;43: 101-109.

11 Pogarell O, Koch W, Pöpperl G, Tatsch K, Jakob F, Zwanzger P, et al: Striatal dopamine release after prefrontal repetitive transcranial magnetic stimulation in major depression: preliminary results of a dynamic [123I] IBZM SPECT study. J Psychiatr Res 2006;40: 307-314.

12 Vanderhasselt MA, De Raedt R, Leyman L, Baeken C: Acute effects of repetitive transcranial magnetic stimulation on attentional control are related to antidepressant outcomes. J Psychiatry Neurosci 2009;34:119126. 
13 Vanderhasselt MA, De Raedt R, Baeken C, Leyman L, D'Haenen H: A single session of rTMS over the left dorsolateral prefrontal cortex influences attentional control in depressed patients. World J Biol Psychiatry 2009; 10:34-42.

- 14 Hoeppner J, Schulz M, Irmisch G, Mau R, Schläfke D, Richter J: Antidepressant efficacy of two different rTMS procedures. High frequency over left versus low frequency over right prefrontal cortex compared with sham stimulation. Eur Arch Psychiatry Clin Neurosci 2003;253:103-109.

- 15 Hoeppner J, Padberg F, Domes G, Zinke A, Herpertz SC, Grossheinrich N, et al: Influence of repetitive transcranial magnetic stimulation on psychomotor symptoms in major depression. Eur Arch Psychiatry Clin Neurosci 2010;260:197-202.

- 16 Baeken C, De Raedt R, Santermans L, Zeeuws D, Vanderhasselt MA, Meers M, et al: HF-rTMS treatment decreases psychomotor retardation in medication-resistant melancholic depression. Prog Neuropsychopharmacol Biol Psychiatry 2010;30:684-687.

- 17 Sabbe B, Hulstijn W, Van Hoof J, Zitman F: Fine motor retardation and depression. J Psychiatr Res 1996;30:295-306.

18 Sabbe B, Hulstijn W, van Hoof J, TuynmanQua HG, Zitman F: Retardation in depression: assessment by means of simple motor tasks. J Affect Disord 1999;55:39-44.

$\checkmark 19$ Pier MP, Hulstijn W, Sabbe BG: Differential patterns of psychomotor functioning in unmedicated melancholic and nonmelancholic depressed patients. J Psychiatr Res 2004a;38: 425-435.
20 Baeken C, Schrijvers DL, Sabbe BG, Vanderhasselt MA, De Raedt R: Impact of one HFrTMS session on fine motor function in right-handed healthy female subjects: a comparison of stimulation over the left versus the right dorsolateral prefrontal cortex. Neuropsychobiology 2012;65:96-102.

21 Sheehan DV, Lecrubier Y, Sheehan KH, Amorim P, Janavs J, Weiller E, et al: The Mini- International Neuropsychiatric Interview (M.I.N.I.): the development and validation of a structured diagnostic psychiatric interview for DSM-IV and ICD-10. J Clin Psychiatry 1998;20:22-57.

22 Beck AT, Ward CH, Mendelson M, Mock J, Erbaugh J: An inventory for measuring depression. Arch Gen Psychiatry 1961;4:561571.

23 Hamilton M: A rating scale for depression. J Neurol Neurosurg Psychiatry 1960;23:56-62.

24 Thase ME, Rush AJ: Treatment-resistant depression; in Bloom FE, Kupfer DJ (eds): Psychopharmacology: the Fourth Generation of Progress. Raven Press, New York, 1995, pp 1081-1097.

25 Rush AJ, Thase ME, Dube S: Research issues in the study of difficult-to-treat depression. Biol Psychiatry 2003;53:743-753.

26 Schrijvers D, De Bruijn ERA, Maas Y, De Grave C, Sabbe BGC, Hulstijn W: Action monitoring in major depressive disorder with psychomotor retardation. Cortex 2008; 44:569-579.

27 Van Strien JW: Handvoorkeur en taaldominantie. Neuropraxis 2001b; 2:10-15.

28 Peleman K, Van Schuerbeek P, Luypaert R, Stadnik T, De Raedt R, De Mey J, et al: Using 3D-MRI to localize the dorsolateral prefrontal cortex in TMS research. World J Biol Psychiatry 2010;11:425-430.

-29 Wassermann EM: Risk and safety of repetitive transcranial magnetic stimulation: report and suggested guidelines presented at the International Workshop on the Safety of Repetitive Transcranial Magnetic Stimulation, June 5-7, 1996. Electroencephalogr Clin Neurophysiol 1998;108:1-16.
30 Rossi S, Hallett M, Rossini PM, Pascual-Leone A, Safety of TMS Consensus Group: Safety, ethical considerations, and application guidelines for the use of transcranial magnetic stimulation in clinical practice and research. Clin Neurophysiol 2009;120:20082039.

31 Pridmore S, Bruno R, Turnier-Shea Y, Reid P, Rybak M: Comparison of unlimited numbers of rapid transcranial magnetic stimulation and ECT treatment sessions in major depressive episode. Int J Neuropsychopharmacol 2000;3:129-134.

-32 Kaptchuk TJ, Goldman P, Stone DA, Stason WB: Do medical devices have enhanced placebo effects? J Clin Epidemiol 2000;53:786792.

33 Loo C, Sachdev P, Elsayed H, McDarmont B, Mitchell P, Wilkinson M, et al: Effects of a 2- to 4-week course of repetitive transcranial magnetic stimulation (rTMS) on neuropsychologic functioning, electroencephalogram, and auditory threshold in depressed patients. Biol Psychiatry 2001;49:615-623.

>34 McDonald WM, Durkalski V, Ball ER, Holtzheimer PE, Pavlicova M, Lisanby SH, Avery D, Anderson BS, Nahas Z, Zarkowski P, Sackeim HA, George MS: Improving the antidepressant efficacy of transcranial magnetic stimulation: maximizing the number of stimulations and treatment location in treatment-resistant depression. Depress Anxiety 2011;28:973-980.

35 George MS, Post RM: Daily left prefrontal repetitive transcranial magnetic stimulation for acute treatment of medication-resistant depression. Am J Psychiatry 2011;168:356364. 